\title{
Perinatal outcomes of human singletons conceived naturally versus assisted reproductive technologies: analysis of the effect of stimulated IVF, modified natural IVF, and frozen embryo transfer
}

\author{
Adhwaa Khudhari ${ }^{1 *}$ (D, Ali Mourad ${ }^{1,2}$, Simon Phillips ${ }^{1,3}$, Mohammad Zubair Alam $^{4}$, Robert Hemmings ${ }^{5}$ and
} Wael Jamal ${ }^{1,2,6}$

\begin{abstract}
Background: Obstetrical outcomes in assisted reproduction techniques (ART) were compared with naturally conceived pregnancies and among each other in multiple reports. However, many important changes in the practice of in vitro fertilization (IVF) over the years, including single embryo transfers (sET) and the introduction of modified natural IVF (mnIVF), and the advances in the frozen embryo transfer (FET) might have impacted the outcomes. Our study is the first to our knowledge to assess four different groups, including spontaneous pregnancies, mnIVF, stimulated IVF (sIVF), and FET altogether in a head-to-head comparison. This is a retrospective study on perinatal outcomes of singleton babies conceived naturally or using three different ART protocols between 2011 and 2014. The primary objective was the comparison of gestational age and birth weight between spontaneously conceived pregnancies (NAT, $n=15,770)$, mnIVF $(n=235)$, IVF $(n=389)$, and FET ( $n=222)$.

Results: Our results show a significant difference in favor of naturally conceived pregnancies over ART in term of gestational age. In fact, the gestational age of babies in the NAT group was statistically higher compared to each one of the ART groups alone. Regarding the birth weight, the mean was significantly higher in the FET group compared to the other categories.

Conclusion: Differences in perinatal outcomes are still found among babies born after different modes of conception. However, there is still need for well-designed high-quality trials assessing perinatal outcomes between naturally conceived pregnancies and different ART protocols based on different maternal and treatment characteristics.
\end{abstract}

Keywords: Perinatal outcome, Singleton, ART, Gestational age, Birth weight

\footnotetext{
* Correspondence: dr.akhudari@yahoo.com

'OVO Fertilité Clinique, 8000 Boulevard Décarie, Montréal, Québec, Canada

Full list of author information is available at the end of the article
}

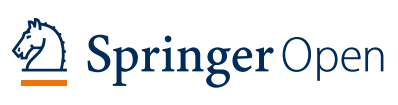

(c) The Author(s). 2021 Open Access This article is licensed under a Creative Commons Attribution 4.0 International License, which permits use, sharing, adaptation, distribution and reproduction in any medium or format, as long as you give appropriate credit to the original author(s) and the source, provide a link to the Creative Commons licence, and indicate if changes were made. The images or other third party material in this article are included in the article's Creative Commons licence, unless indicated otherwise in a credit line to the material. If material is not included in the article's Creative Commons licence and your intended use is not permitted by statutory regulation or exceeds the permitted use, you will need to obtain permission directly from the copyright holder. To view a copy of this licence, visit http://creativecommons.org/licenses/by/4.0/. 


\section{Background}

The ultimate objective of assisted reproductive technologies (ART) is to efficiently achieve healthy live births. It has been observed that various processes and procedures associated with ART may increase the risk of adverse perinatal outcomes $[1,2]$. Since the birth of Louise Brown in 1978 until 2008, the world has seen more than 6 million babies conceived through different IVF [3]. While the majority of these IVF babies appeared to be healthy, a higher incidence of some congenital abnormalities, preterm birth, and small for gestational age were observed among ART singletons [4]. The latter problem is of increasing concern since birth weight is an indicator for fetal growth and a strong predictor of cardio-metabolic risks in long-term follow-ups $[5,6]$. Literature also suggests an association between IVF and neurodevelopmental disorders as well as potentially long-term metabolic outcomes [7, 8]. Apart from these, it was found that infertility characteristics of the parents may also influence perinatal outcomes [9]. Several other studies have shown ART pregnancies to be associated with a higher risk of complications as compared to spontaneously conceived pregnancies [9-11].

Interestingly, many previous studies have shown that children born after FET had a higher birth weight and fewer adverse perinatal outcomes as compared to children born after fresh cycles [11, 12 13]. Moreover, FET singletons have perinatal outcomes comparable with those observed in naturally conceived singletons [13, 14]. The more natural endometrial preparation leading to a natural placentation in these cycles seems to play a crucial role in these findings [15].

When it comes to the modified natural IVF cycles, it was found to be a reasonable option for women around 35 years of age as it minimizes physical and emotional stress for the patient, significantly reduces the cost of drugs and laboratory tests for the assisted reproduction unit, decreases the chances of multiple pregnancy and ovarian hyper-stimulation, and eliminates the need for the patient to go through a resting cycle [16-18]. This more natural environment in a modified natural IVF cycle can explain as well the reason for a higher birth weight in these cycles when compared to standard stimulated IVF [19].

The underlying etiologies of these adverse perinatal outcomes that were observed in ART are largely unknown, but contributing factors may include the subfertility of the couple, use of hormonal stimulation, and the use of various ART techniques [9, 20]. Moreover, maternal age, smoking, high BMI, and the duration of infertility have also been reported to increase the risk of low birth weight [21-23]. Even in the same mother, ART singletons had a poorer outcome as compared to their non-ART-conceived siblings [9].
The present study was done to compare the perinatal outcomes of singleton babies conceived through three different ART protocols and through natural conception.

\section{Methods}

All naturally conceived liveborn singletons delivered at St. Mary's Community Hospital in Montreal, Canada, and liveborn singletons resulting from IVF treatment at the university affiliated fertility center OVO clinic in Montreal, Canada, between 2010 and 2014 were selected. A total number of 20,713 deliveries were evaluated, among them 16,616 cases met the inclusion criteria and were incorporated in the analysis.

The inclusion criteria comprised of the following conditions: maternal age of 41 years and below, gestational age at the time of delivery of 20 weeks and above, birth weight of 600 grams and above, and a BMI of $35 \mathrm{~kg} / \mathrm{m}^{2}$ and below. Only patients who received a sET were included, whereas women who received more than one embryo per transfer were excluded. Moreover, multiple births were excluded from the analysis.

The births were distributed according to the mode of conception. Thus, patients were categorized into four groups: (group 1) babies conceived naturally without any medical assistance in normal fertile couples (NAT, $n=15$, 770); (group 2) babies conceived through mnIVF ( $n=$ 235); (group 3) babies conceived sIVF $(n=389)$; and (group 4) babies conceived through IVF but only following FET $(n=222)$. In the previously mentioned ART protocols, embryos were transferred on day 2,3 , or 5 post-fertilization.

After obtaining the approval of the scientific-ethical committee at OVO clinic as a quality control, data were retrospectively retrieved between 2010 and 2014. A chart review of the electronic and paper records of all included cases was performed. Demographic information including maternal age, infertility type (primary, secondary), years of infertility, BMI, ovarian reserve, AMH levels, smoking status; treatment variables including ART protocol (mnIVF, sIVF, FET), insemination type (conventional IVF, ICSI), type of embryo, the day of embryo transfer; and perinatal outcomes including gestational age, birth weight, sex of the baby, and mode of delivery (cesarean, vaginal) were collected.

The modified natural IVF protocol consisted of monitoring follicular development with trans-vaginal ultrasound. When the leading follicle reached a diameter of $15 \mathrm{~mm}$ with an endometrial thickness $\geq 6 \mathrm{~mm}$, human menopausal gonadotropin (hMG) injections (Menopur or Repronex, Ferring Pharmaceuticals, Canada) at a dose of $150 \mathrm{IU}$ daily along with indomethacin $50 \mathrm{mg}$ orally three times daily and GnRH antagonist Orgalotran 250 mcg injection daily (Merck pharmaceutical, Canada) were started until the day of trigger of final oocyte 
maturation. Follow-up ultrasound was performed accordingly. When the leading follicle reached a diameter between 17 and $20 \mathrm{~mm}$, estradiol and progesterone dosages were performed for possible cancelation and hCG triggering using 5000 IU followed by ovum pick up $34 \mathrm{~h}$ later on was conducted.

Stimulated IVF cycles were performed using standard $\mathrm{GnRH}$ antagonist, long agonist, or micro-dose flare protocols. Follicular development and estradiol levels were monitored during the stimulation and on the day of trigger. When 3 follicles measuring $\geq 18 \mathrm{~mm}$ were obtained, hCG triggering was administered. Oocyte retrieval was performed $36 \mathrm{~h}$ after triggering of final oocyte maturation. ICSI was performed for male factor, unexplained infertility, prior fertilization failure with conventional insemination, preimplantation genetic testing (PGT), poor-quality oocytes, low oocyte yield, and advanced maternal age. Fresh embryo transfer was performed if the risk of ovarian hyper-stimulation syndrome (OHSS) was deemed low.

For the FET cycles, endometrial preparation was carried out using different protocols based on the clinical background of patients, their prior response if available, and the discretion of the physician. The protocols included either FET in a natural cycle, hormone replacement conventional substituted, or stimulated.

The gestational age at the time of delivery and birth weight were considered the main outcomes, whereas the sex of the baby and the mode of delivery were considered as secondary outcomes. Obstetrical complications were not covered by this study.

Statistical analyses were performed using the SAS/ STAT $^{\circ}$ software (SAS University Edition, version 9.4M5; SAS Institute Inc. Cary NC, USA). Data were tested for normality by Kolmogorov-Smirnov test and transformed to natural logarithms or ranks as appropriate. NAT, mnIVF, sIVF, and FET were compared for the demographic information, the treatment variables and the perinatal outcomes previously mentioned.

In order to determine the relationship between each one of the a foure mentioned groups, as explanatory variables, and perinatal outcomes as dependent variables, a univariate analysis was performed. Chi-square test was performed for categorical variables, whereas Student's $t$ test was performed for continuous variables. A $p$ value $<$ 0.05 was considered as statistically significant in all tests. Results were presented as mean \pm standard deviation (SD) or as percentages. Moreover, a head-to-head comparison was performed between each two groups alone for both primary outcomes. Results for the multiple comparison were presented as mean difference with $95 \%$ confidence interval and a $p$ value.

To control for potentially confounding variables, conception type, years of infertility, type of insemination, smoking status, AMH level, category of BMI, type of infertility, ovarian reserve, day of transfer, BMI, and maternal age were included in a backward regression analysis in the ART groups for gestational age. Similarly, the same variables in addition to the gestational age were included in a backward regression analysis in the ART groups for birth weight. The selection method in this analysis consists of entering the independent variables into the equation first and each factor is then deleted one at a time if they do not contribute to the regression equation. The strength for the effect size in the backward regression analysis was evaluated using the $R$-squared value.

\section{Results}

Regarding the demographic characteristics of included patients, there was a statistically significant difference in terms of maternal age $(p<0.0001)$. Patients who conceived naturally were younger compared to patients who conceived through ART. For instance, the mean age for the NAT group was 30.69 years \pm 4.34 versus $38.78 \pm$ $3.12,38.63 \pm 3.46$, and $40.39 \pm 2.06$ in the mnIVF, sIVF, and FET respectively (Table 1) This difference was expected since patients who present to fertility clinics are usually older compared to normal fertile couples, as detailed in the "Discussion" section. Interestingly, there was no significant difference for the maternal age among various ART groups. Regarding AMH level, there are no data for the NAT group; however, there was a statistical difference between the ART groups $(p<0.0001)$. In fact, mnIVF had the lowest AMH $(1.99 \mathrm{ng} / \mathrm{ml} \pm 2.62)$ followed by $\operatorname{sIVF}(2.72 \mathrm{ng} / \mathrm{ml} \pm 2.69)$ and then FET (3.61 $\mathrm{ng} / \mathrm{ml} \pm 4.76$ ) (Table 1 ). This can be explained by the indication for mnIVF which includes patients with decreased ovarian reserve and premature ovarian failure; hence, the AMH is expected to be lower in this group. On the other hand, patients who obtain multiple embryos are more likely to have frozen embryos, and thus, more likely to have a better ovarian reserve to start with, which can explain the higher level of AMH in the FET group. In the multiple regression analysis for both main outcomes: birth weight and gestational age, AMH was not found to be a significant predictor when compared between the three different ART groups, and hence is not a confounding factor (Supplemental Table 3) (Supplemental Table 6). Likewise, a difference was noted for the antral follicular count $(p<0.0001)$, following a similar pattern of the AMH level, which corresponds well to the ovarian reserve of these patients (Table 1). Regarding maternal age, it was not found to be a predictive factor in the three ART groups in the regression analysis for the birth weight (Supplemental Table 3); however, a significant relationship between maternal age and gestational age was noted in the ART groups 
Table 1 Demographic characteristics of included patients

\begin{tabular}{|c|c|c|c|c|c|c|}
\hline \multicolumn{2}{|c|}{ Characteristics } & NAT & mnIVF & sIVF & FET & $p$ value \\
\hline \multicolumn{2}{|c|}{ No. of women $(n)$} & 15770 & 235 & 384 & 222 & \\
\hline \multicolumn{2}{|l|}{ Age (years) } & $30.69 \pm 4.34$ & $38.78 \pm 3.12$ & $38.63 \pm 3.46$ & $40.39 \pm 2.06$ & $p<0.0001$ \\
\hline \multicolumn{2}{|l|}{ AMH (ng/ml) } & & $1.99 \pm 2.62$ & $2.72 \pm 2.69$ & $3.61 \pm 4.76$ & $p<0.0001$ \\
\hline \multicolumn{2}{|c|}{ Antral follicular Count } & & $17.30 \pm 9.90$ & $21.42 \pm 10.46$ & $22.54 \pm 11.64$ & $p<0.0001$ \\
\hline \multicolumn{2}{|c|}{$\begin{array}{l}\text { Infertility type } \\
\text { Primary/secondary }\end{array}$} & & $154 / 73$ & $230 / 136$ & $129 / 80$ & $p=0.340$ \\
\hline \multicolumn{2}{|c|}{$\begin{array}{l}\text { Years of infertility } \\
1 \text { year/ } \geq 2 \text { years }\end{array}$} & & $134 / 54$ & $228 / 81$ & $117 / 61$ & $p=0.168$ \\
\hline \multirow[t]{4}{*}{ BMI $\left(\mathrm{kg} / \mathrm{m}^{2}\right)$} & $<18.5$ & & 3 & 9 & 8 & $p=0.216$ \\
\hline & $18.5-24.9$ & & 110 & 118 & 80 & $p=0.123$ \\
\hline & $25.0-29.9$ & & 44 & 49 & 42 & $p=0.598$ \\
\hline & $30.0-34.9$ & & 19 & 40 & 24 & $p=0.105$ \\
\hline \multicolumn{3}{|c|}{ Smoking/non-smoking } & $21 / 121$ & $29 / 166$ & $17 / 104$ & $p=0.978$ \\
\hline \multicolumn{2}{|c|}{$\begin{array}{l}\text { Insemination type } \\
\text { Conventional IVF/ICSI }\end{array}$} & & $112 / 126$ & $115 / 223$ & & $p=0.002$ \\
\hline
\end{tabular}

Results are presented as mean \pm SD or ratio

(Supplemental Table 6). As for the type of insemination (conventional IVF versus ICSI), two groups were only compared: mnIVF and sIVF. In order to prevent absence of fertilization on a single oocyte in the mnIVF group, ICSI was performed in the vast majority of these patients (88.9\%), which is not the case of the mnIVF where ICSI was performed based on the aforementioned indications in the "Methods" section. Thus, a significant difference was also noted when comparing both groups for the type of insemination ( $p=0.002)$ (Table 1). In the multiple regression analysis for both main outcomes: birth weight and gestational age, the type of insemination was not found to be a significant predictor when compared between the mnIVF and sIVF (Supplemental Table 3) (Supplemental Table 6). Concerning the type of infertility, the years of infertility, smoking status, and the category of BMI, no statistical difference was noted between the ART groups. Data were either not applicable or not available for the NAT group regarding the previously mentioned characteristics except for the maternal age.

With respect to the perinatal outcomes, a significant difference was noted in terms of gestational age at the time of delivery $(p<0.0001)$. NAT group had the highest gestational age (39.13 weeks \pm 1.41$)$ compared to the ART groups that manifested a slightly younger gestational age: around 38 weeks $(38.37 \pm 2.15,38.11 \pm 2.20$, and $38.57 \pm 1.72$ for mnIVF, sIVF, and FET respectively) (Table 2). It is worth mentioning that the mean gestational age at the time of delivery for all groups reached term (above 37 weeks). A head-to-head comparison between all groups was performed for the gestational age. In fact, the gestational age of babies conceived naturally was statistically higher compared to each one of the ART groups alone (Table 3). No difference was noted when comparing mnIVF to sIVF and mnIVF to FET; however, a statistically higher gestational age was noted in the FET group compared to sIVF (Table 3).

Regarding the birth weight, the mean was significantly higher in the FET group compared to the other categories with an average of 3444.9 grams $\pm 576.7(p<0.0001)$ (Table 2). A head-to-head comparison between all groups was performed for the birth weight as well. In fact, the birth weight of babies conceived through FET naturally was statistically higher than all other groups compared (Table 4). However, no difference was noted between mnIVF and sIVF or between NAT and mnIVF (Table 4).

As for the sex ratio, it was in favor of male babies in NAT and sIVF groups whereas female babies were more predominant in the mnIVF and FET groups $(p<0.05)$

Table 2 Perinatal outcomes of included patients

\begin{tabular}{llllll}
\hline Outcomes & NAT & mnIVF & sIVF & FET & p value \\
\hline Gestation age (weeks) & $39.13 \pm 1.41$ & $38.37 \pm 2.15$ & $38.11 \pm 2.20$ & $38.57 \pm 1.72$ & $p<0.0001$ \\
Birth weight (grams) & $3351.2 \pm 482.1$ & $3301.2 \pm 38.8$ & $3257.0 \pm 571.4$ & $3444.9 \pm 576.7$ & $p<0.0001$ \\
Sex ratio (male/female) & $1.06(10231 / 9619)$ & $0.82(106 / 130)$ & $1.23(209 / 170)$ & $0.89(101 / 113)$ & $p<0.05$ \\
Delivery (cesarean/natural) & $0.33(4921 / 14931)$ & $0.44(70 / 160)$ & $0.38(103 / 274)$ & $0.53(72 / 136)$ & $p<0.0001$ \\
\hline
\end{tabular}

Results are presented as mean \pm SD 
Table 3 Multiple comparisons of gestational age between different modes of conception

\begin{tabular}{lllllll}
\hline $\begin{array}{l}\text { Mode of } \\
\text { conception (1) }\end{array}$ & $\begin{array}{l}\text { Mode of } \\
\text { conception (2) }\end{array}$ & $\begin{array}{l}\text { Mean } \\
\text { difference (1-2) }\end{array}$ & Std. error & $\boldsymbol{p}$ value & & \multicolumn{2}{c}{$\mathbf{9 5 \% \text { Confidence interval }}$} \\
\hline NAT & mnIVF & 0.7068 & 0.10580 & $<0.0001$ & 0.4276 & Upper bound \\
NAT & SIVF & 1.0241 & 0.08257 & $<0.0001$ & 0.8062 & 0.9860 \\
NAT & FET & 0.5688 & 0.10894 & $<.00001$ & 0.2814 & 0.2419 \\
mnIVF & SIVF & 0.3173 & 0.13294 & 0.102 & -0.0335 & 0.8563 \\
mnIVF & FET & -0.1379 & 0.15074 & 1.000 & -0.5357 & 0.6681 \\
SIVF & FET & -0.4552 & 0.13545 & 0.005 & -0.8126 & -0.0978 \\
\hline
\end{tabular}

(Table 2). Even though vaginal deliveries were more prevalent in all groups compared to cesarean sections, however, a higher ratio of cesarean/vaginal deliveries was noted in the FET group, interestingly the same group with the highest mean birth weight.

In order to adjust for possible confounding factors, a backward regression analysis was performed for the three ART groups (mnIVF, sIVF, FET). All the characteristics were included in the analysis for gestational age, and the same variables in addition to the gestational age were included in the analysis for birth weight. The result shows no linear relationship between the variables, except for a clear correlation $(p=0.0002)$ between gestational age and birth weight, which is expected since birth weight is obviously dependent on the gestational age. For all of the obtained backward regression models, the $R$-squared value is smaller than 0.5 (Supplemental Table 1) or less than 0.1 (Supplemental Table 2). In general, an $R$-squared value between 0.5 and 0.7 means there is a moderate effect size, while an $R$-squared value $>0.7$ is considered to indicate a strong effect size. Hence, in this analysis, it seems that there is a weak and even very weak effect size for the obtained backward regression models. In conclusion, the studied variables have no or little effect on the measured outcomes, supporting the evidence of the study results.

\section{Discussion}

Performance of ART changed significantly over the years with all the technological advances and accumulated experience. In parallel, notable adjustment and adaptation of perinatal care remarkably influenced neonatal outcomes [24]. This dual progress, hence, creates a need for a contemporary and an updated evaluation of the outcome of pregnancies of different IVF protocols. Our study was performed between 2010 and 2014, which can be considered as relatively new in comparison to older studies evaluating the same subject of interest $[25,26]$. Moreover, most perinatal complications of IVFconceived pregnancies are related to the high incidence of multiple gestations [27]. This does not apply to the current practice of ART, where single embryo transfers have become the standard of care in multiple countries and fertility centers [28]. One of the strengths of our study is the exclusive evaluation of singleton pregnancies, in order to suit the modern trend in ART. Furthermore, the introduction of less invasive and more natural performance in current IVF protocols such as mnIVF and FET has positively impacted the obstetrical outcomes. For instance, the reasonably natural stimulation in mnIVF was postulated to be the reason for a higher birth weight in these cycles when compared to standard sIVF [19]. Likewise, FET singletons were found to have similar perinatal outcomes comparable with those observed in naturally conceived pregnancies [29, 30]. The more natural endometrial environment leading to a natural placentation in these cycles can explain these findings [15]. In the literature, most studies compared perinatal outcomes of spontaneously conceived pregnancies to a single type of IVF protocol, or of two different

Table 4 Multiple comparisons of birth weight between different modes of conception

\begin{tabular}{lllllll}
\hline $\begin{array}{l}\text { Mode of } \\
\text { conception (1) }\end{array}$ & $\begin{array}{l}\text { Mode of } \\
\text { conception (2) }\end{array}$ & $\begin{array}{l}\text { Mean } \\
\text { difference (1-2) }\end{array}$ & Std. error & $\boldsymbol{p}$ value & & \multicolumn{2}{c}{$\mathbf{9 5 \% \text { Confidence interval }}$} \\
\hline NAT & mnIVF & 50.07 & 33.090 & 0.782 & -37.24 & Upper bound \\
NAT & SIVF & 94.38 & 25.843 & 0.002 & 26.19 & 137.38 \\
NAT & FET & -92.77 & 34.031 & 0.038 & -182.56 & -2.98 \\
mnIVF & SIVF & 44.31 & 41.601 & 1.000 & -65.46 & 154.08 \\
mnIVF & FET & -142.84 & 47.127 & 0.015 & -267.19 & -18.49 \\
SIVF & FET & -187.15 & 42.353 & $<0.0001$ & -298.90 & -75.40 \\
\hline
\end{tabular}


ART modalities [19, 29, 31-36]. However, our study is the first to our knowledge to assess all these different groups altogether in a head-to-head comparison, for we included the four main groups that must be evaluated in order to draw specific conclusions for the particular modality in question. The four groups incorporated in our analysis are NAT, mnIVF, sIVF, and FET.

One of the weaknesses of our study remains in the retrospective nature of the data collected. Unfortunately, in the NAT group, consisting of patients who conceived naturally without the need for fertility treatments, many variables were not documented in their charts (AMH, smoking, $\mathrm{BMI}$ ), making the matching process of patients in these different groups impossible. These variables could be possible confounding factors that were not adjusted. However, it is worth mentioning that previous reports found an effect of pre-pregnancy BMI and smoking status on adverse obstetrical outcomes; however, no difference was noted when comparing ART and spontaneously conceived pregnancies who were matched for the variables [37-39].

Our results show a significant difference in favor of naturally conceived pregnancies over ART in term of gestational age. In fact, the gestational age of babies in the NAT group was statistically higher compared to each one of the ART groups alone. These findings are consistent with the report of Woo and his colleagues who analyzed 494 pregnancies (312 spontaneous and 182 surrogates), and found that babies born from ART had lower mean gestational age and higher rates of preterm birth [34]. Regarding the birth weight, the mean was significantly higher in the FET group compared to the other categories. This finding was reproduced in many other reports studying birth weight in FET cycles. For instance, a higher birth weight and fewer adverse perinatal outcomes as compared to babies born after fresh ETs in sIVF were reported $[29,30]$. The latter reproducible finding was mainly attributed to a more natural placentation process in FET cycles [15]. One additional interesting finding in our report is the higher maternal age that was found in the ART groups compared to NAT, and this can be attributed mainly to the higher incidence of older women seeking fertility treatments [40].

In conclusion, the following study shows perinatal outcomes differ based on the mode of conception. The results are consistent with other studies showing a lower average gestational age at delivery among ART babies compared to naturally conceived pregnancies and a higher average live birth weight in FET singleton babies compared to all other groups. Further studies should be performed, listing all the possible maternal and treatment variables that might have an effect on obstetrical outcomes in the different ART groups and naturally conceived pregnancies, in order to generate a model able to predict the gestational age and birth weight in terms of these predictors.

\section{Supplementary Information}

The online version contains supplementary material available at https://doi. org/10.1186/s43043-021-00051-w.

Additional file 1: Supplemental Table 1. Model summary: regression analysis including ART only groups for birth weight. Supplemental Table 2. Regression model characteristics in the ART groups only for birth weight. Supplemental Table 3. Detailed regression model including ART groups only for birth weight by predictor. Supplemental Table 4. Model summary: regression analysis including ART only groups for gestational age. Supplemental Table 5. Regression model characteristics in the ART groups only for gestational age. Supplemental Table 6. Detailed regression model including ART groups only for gestational age by predictor. Supplemental Table 1. Backward regression analysis including ART groups for birth weight. Supplemental Table 2. Backward regression model analysis including ART groups for gestational age

\begin{abstract}
Abbreviations
AMH: Anti-mullerian hormone; BMI: Body mass index; E2: Estradiol; FET: Frozen embryo transfer; HCG: Human chorionic gonadotropin; HMG: Human menopausal gonadotropin; ICSI: Intracytoplasmic sperm injection; IRB: Institutional review board; IVF: In vitro fertilization; sIVF: Stimulated IVF; GnRH: Gonadotropin releasing hormone; mnIVF: Modified natural in vitro fertilization; LBW: Low birth weight; ART: Assisted reproduction technologies; sET: Single embryo transfer; OHSS: Ovarian hyper-stimulation syndrome; PGT: Pre-implantation genetic testing
\end{abstract}

Acknowledgements

The authors are grateful to Dr. Mourad and Dr. Zubair for participating in the study.

Authors' contributions

AK (data collection, writing the manuscript, final editing), WJ (research concept, manuscript editing, data review), AM (writing the manuscript, final editing), RH (data analysis, final editing), SP (data review, manuscript editing, final review), MZ (data analysis, wiring manuscript). All authors have read and approved the manuscript.

Funding

No funding was obtained for this study.

Availability of data and materials

The datasets generated and/or analyzed during the current study are not publicly available due to patients' information but are available from the corresponding author on reasonable request.

Ethics approval and consent to participate

Ethics and Scientific committee at OVO clinic at Montreal, Canada, approval was obtained for this study and no consent was required for the data collection in this retrospective study.

\section{Consent for publication}

Not applicable.

\section{Competing interests \\ Not applicable}

\section{Author details}

${ }^{1}$ OVO Fertilité Clinique, 8000 Boulevard Décarie, Montréal, Québec, Canada. ${ }^{2}$ Department of Obstetrics and Gynecology, McGill University, Montreal, Quebec, Canada. ${ }^{3}$ Department of Obstetrics and Gynecology, University of Montreal, Montreal, Quebec, Canada. ${ }^{4}$ Faculty of Medicine, University of Montreal, Montreal, Canada. ${ }^{5}$ Faculty of Medicine Rabigh Campus, King Abdul Aziz University, Rabigh, Saudi Arabia. ${ }^{6}$ King Fahd Medical Research Center, King Abdul Aziz University, Jeddah, Saudi Arabia. 
Received: 18 December 2019 Accepted: 14 February 2021 Published online: 06 March 2021

\section{References}

1. Olivennes F, Rufat P, André B, Pourade A, Quiros MC, Frydman R (1993) The increased risk of complication observed in singleton pregnancies resulting from in-vitro fertilization (IVF) does not seem to be related to the IVF method itself. Hum Reprod 8(8):1297-1300

2. Sundström I, Ildgruben A, Högberg U (1997) Treatment-related and treatment-independent deliveries among infertile couples, a long-term follow-up. Acta Obstet Gynecol Scand. 76(3):238-243

3. Hann M, Roberts SA, D'Souza SW, Clayton P, Macklon N, Brison DR. The growth of assisted reproductive treatment-conceived children frombirth to 5 years: a national cohort study. BMC Medicine. 2018;16:224. [cited 2019 May 30].

4. Sunde A, Brison D, Dumoulin J, Harper J, Lundin K, Magli MC et al (2016) Time to take human embryo culture seriously. Hum Reprod 31(10):2174-2182

5. Valenzuela-Alcaraz B, Crispi F, Bijnens B, Cruz-Lemini M, Creus M, Sitges M et al (2013) Assisted reproductive technologies are associated with cardiovascular remodeling in utero that persists postnatally. Circulation 128(13):1442-1450

6. Pontesilli M, Painter RC, Grooten IJ, van der Post JA, Mol BW, Vrijkotte TGM et al (2015) Subfertility and assisted reproduction techniques are associated with poorer cardiometabolic profiles in childhood. Reprod Biomed Online 30(3):258-267

7. Ombelet W, Martens G, Bruckers L (2016) Pregnant after assisted reproduction: a risk pregnancy is born! 18-years perinatal outcome results from a population-based registry in Flanders, Belgium. Facts Views Vis Obgyn 8(4):193-204

8. Sullivan-Pyke CS, Senapati S, Mainigi MA, Barnhart KT (2017) In vitro fertilization and adverse obstetric and perinatal outcomes. Semin Perinatol 41(6):345-353

9. Pinborg A (2013) Large baby syndrome in singletons born after frozen embryo transfer (FET). Hum Reprod 29(3):618-627

10. McDonald SD, Han Z, Mulla S, Murphy KE, Beyene J, Ohlsson A et al (2009) Preterm birth and low birth weight among in vitro fertilization singletons: a systematic review and meta-analyses. Eur J Obstet Gynecol Reprod Biol 146(2):138-148

11. McDonald SD, Han Z, Mulla S, Ohlsson A, Beyene J, Murphy KE et al (2010) Preterm birth and low birth weight among in vitro fertilization twins: a systematic review and meta-analyses. Eur J Obstet Gynecol Reprod Biol 148(2):105-113

12. Pinborg A, Loft A, Aaris Henningsen A-K, Rasmussen S, Andersen AN (2010 Sep) Infant outcome of 957 singletons born after frozen embryo replacement: the Danish National Cohort Study 1995-2006. Fertil Steril 94(4): 1320-1327

13. Pelkonen S, Koivunen R, Gissler M, Nuojua-Huttunen S, Suikkari A-M, HydénGranskog C et al (2010) Perinatal outcome of children born after frozen and fresh embryo transfer: the Finnish cohort study 1995-2006. Hum Reprod 25(4):914-923

14. Sazonova A, Källen K, Thurin-Kjellberg A, Wennerholm U-B, Bergh C (2012) Obstetric outcome in singletons after in vitro fertilization with cryopreserved/thawed embryos. Hum Reprod 27(5):1343-1350

15. Wennerholm U-B, Henningsen A-KA, Romundstad LB, Bergh C, Pinborg A, Skjaerven $\mathrm{R}$ et al (2013) Perinatal outcomes of children born after frozenthawed embryo transfer: a Nordic cohort study from the CoNARTaS group. Hum Reprod 28(9):2545-2553

16. Phillips SJ, Kadoch IJ, Lapensée L, Couturier B, Hemmings R, Bissonnette F (2007) Controlled natural cycle IVF: experience in a world of stimulation. Reprod Biomed Online 14(3):356-359

17. Kadoch IJ, Al-Khaduri M, Phillips SJ, Lapensée L, Couturier B, Hemmings R et al (2008) Spontaneous ovulation rate before oocyte retrieval in modified natural cycle IVF with and without indomethacin. Reprod Biomed Online 16(2):245-249

18. Shaulov T, Vélez MP, Buzaglo K, Phillips SJ, Kadoch IJ (2015 Jul) Outcomes of 1503 cycles of modified natural cycle in vitro fertilization: a single-institution experience. J Assist Reprod Genet 32(7):1043-1048

19. Pelinck $M-J$, Keizer $M H$, Hoek A, Simons AHM, Schelling K, Middelburg K et al (2010) Perinatal outcome in singletons after modified natural cycle IVF and standard IVF with ovarian stimulation. Eur J Obstet Gynecol Reprod Biol 148(1):56-61

20. Mak W, Kondapalli LA, Celia G, Gordon J, DiMattina M, Payson M (2016) Natural cycle IVF reduces the risk of low birthweight infants compared with conventional stimulated IVF. Hum Reprod 31(4):789-794

21. Stojnic J, Radunovic N, Jeremic K, Kotlica BK, Mitrovic M, Tulic I (2013) Perinatal outcome of singleton pregnancies following in vitro fertilization. Clin Exp Obstet Gynecol 40(2):277-283

22. Stojnic J, Radunovic N, Jeremic K, Kotlica BK, Mitrovic M, Tulic I. Perinatal outcome of singleton pregnancies following in vitro fertilization. Clin Exp Obstetatrics Gynecol. 2013;40(2):277-83.

23. Berntsen $S$, Söderström-Anttila $V$, Wennerholm U-B, Laivuori H, Loft A, Oldereid NB et al (2019) The health of children conceived by ART: 'the chicken or the egg?'. Hum Reprod Update 25(2):137-158

24. Allen VM, Wilson RD, Cheung A (2006) Genetics committee, reproductive endocrinology and infertility committee. Pregnancy outcomes after assisted reproductive technology. J Obstet Gynaecol Can 28(3):220-233

25. Helmerhorst FM, Perquin DAM, Donker D, Keirse MJNC (2004) Perinatal outcome of singletons and twins after assisted conception: a systematic review of controlled studies. BMJ 328(7434):261

26. Jackson RA, Gibson KA, Wu YW, Croughan MS (2004) Perinatal outcomes in singletons following in vitro fertilization: a meta-analysis. Obstet Gynecol 103(3):551-563

27. American College of Obstetricians and Gynecologists' Committee on Obstetric Practice, Committee on Genetics, U.S. Food and Drug Administration (2016) Committee opinion no 671: perinatal risks associated with assisted reproductive technology. Obstet Gynecol 128(3):e61-e68

28. Practice Committee of American Society for Reproductive Medicine (2012) Elective single-embryo transfer. Fertil Steril 97(4):835-842

29. Pelkonen S, Koivunen R, Gissler M, Nuojua-Huttunen S, Suikkari A-M, HydénGranskog C et al (2010) Perinatal outcome of children born after frozen and fresh embryo transfer: the Finnish cohort study 1995-2006. Hum Reprod 25(4):914-923

30. Sazonova A, Källen K, Thurin-Kjellberg A, Wennerholm U-B, Bergh C (2012) Obstetric outcome in singletons after in vitro fertilization with cryopreserved/thawed embryos. Hum Reprod 27(5):1343-1350

31. Sullivan-Pyke CS, Senapati S, Mainigi MA, Barnhart KT (2017) In vitro fertilization and adverse obstetric and perinatal outcomes. Semin Perinato 41(6):345-353

32. Dhont M, De Sutter P, Ruyssinck G, Martens G, Bekaert A (1999) Perinatal outcome of pregnancies after assisted reproduction: a case-control study. Am J Obstet Gynecol 181(3):688-695

33. Pandey S, Shetty A, Hamilton M, Bhattacharya S, Maheshwari A (2012) Obstetric and perinatal outcomes in singleton pregnancies resulting from IVF/ICSI: a systematic review and meta-analysis. Hum Reprod Update 18(5):485-503

34. Woo I, Hindoyan R, Landay M, Ho J, Ingles SA, McGinnis LK et al (2017) Perinatal outcomes after natural conception versus in vitro fertilization (IVF) in gestational surrogates: a model to evaluate IVF treatment versus maternal effects. Fertil Steril 108(6):993-998

35. Storgaard M, Loft A, Bergh C, Wennerholm UB, Söderström-Anttila V, Romundstad LB et al (2017) Obstetric and neonatal complications in pregnancies conceived after oocyte donation: a systematic review and meta-analysis. BJOG 124(4):561-572

36. Spijkers S, Lens JW, Schats R, Lambalk CB (2017) Fresh and frozen-thawed embryo transfer compared to natural conception: differences in perinatal outcome. Gynecol Obstet Invest 82(6):538-546

37. Farhi A, Reichman B, Boyko V, Hourvitz A, Ron-El R, Lerner-Geva L (2013) Maternal and neonatal health outcomes following assisted reproduction. Reprod Biomed Online 26(5):454-461

38. Korsholm A-S, Petersen KB, Bentzen JG, Hilsted LM, Andersen AN, Hvidman HW (2018) Investigation of anti-Müllerian hormone concentrations in relation to natural conception rate and time to pregnancy. Reprod Biomed Online 36(5):568-575

39. Frankenthal D, Hirsh-Yechezkel G, Boyko V, Orvieto R, Ron-El R, Lerner-Geva $L$ et al (2019) The effect of body mass index (BMI) and gestational weight gain on adverse obstetrical outcomes in pregnancies following assisted reproductive technology as compared to spontaneously conceived pregnancies. Obes Res Clin Pract 13(2):150-155 
40. Gleicher N, Kushnir VA, Albertini DF, Barad DH (2016) Improvements in IVF in women of advanced age. J Endocrinol 230(1):F1-F6

41. Veleva Z, Orava M, Nuojua-Huttunen S, Tapanainen JS, Martikainen H (2013 Sep) Factors affecting the outcome of frozen-thawed embryo transfer. Hum Reprod. 28(9):2425-2431

\section{Publisher's Note}

Springer Nature remains neutral with regard to jurisdictional claims in published maps and institutional affiliations.

Submit your manuscript to a SpringerOpen ${ }^{\odot}$ journal and benefit from:

- Convenient online submission

- Rigorous peer review

- Open access: articles freely available online

- High visibility within the field

- Retaining the copyright to your article

Submit your next manuscript at $\boldsymbol{\nabla}$ springeropen.com 